\title{
GAIA SPECTROSCOPY: PROCESSING, PERFORMANCES AND SCIENTIFIC RETURNS
}

D. Katz ${ }^{1}$, M. Cropper ${ }^{2}$, F. Meynadier ${ }^{1}$, A. Jean-Antoine ${ }^{3}$, C. Allende Prieto $^{4}$, S. Baker ${ }^{2}$, K. Benson ${ }^{2}$, J. Berthier ${ }^{5}$, L. Bigot ${ }^{6}$, R. Blomme ${ }^{7}$, S. Boudreault ${ }^{2}$, L. Chemin ${ }^{8}$, F. Crifo ${ }^{1}$, Y. Damerdji ${ }^{9}$, M. David ${ }^{10}$, P. David ${ }^{5}$, C. Delle Luche ${ }^{1}$, C. Dolding ${ }^{2}$, Y. Frémat ${ }^{7}$, N. Gerbier ${ }^{3}$, J. Gerssen ${ }^{11}$, A. Gómez ${ }^{1}$, E. Gosset ${ }^{9}$, A. Guerrier ${ }^{3}$, L. Guy ${ }^{12}$, D. Hall ${ }^{13}$, D. Hestroffer ${ }^{5}$, H. Huckle ${ }^{2}$, G. Jasniewicz ${ }^{14}$, H.-G. Ludwig ${ }^{15}$, C. Martayan ${ }^{1}$, T. Morel $^{9}$, A.-T. Nguyen ${ }^{3}$, P. Ocvirk ${ }^{11}$, C. Parr ${ }^{2}$, F. Royer ${ }^{1}$, P. Sartoretti ${ }^{1}$, G. Seabroke ${ }^{2}$, E. Simon ${ }^{3}$, M. Smith ${ }^{2}$, C. Soubiran ${ }^{8}$, M. Steinmetz ${ }^{11}$, F. Thévenin ${ }^{6}$, C. Turon ${ }^{1}$, S. Udry ${ }^{12}$, L. Veltz ${ }^{11}$ and Y. Viala ${ }^{1}$

\footnotetext{
${ }^{1}$ GÉPI, Observatoire de Paris, CNRS, Université Paris Diderot, 5 place Jules Janssen, 92190 Meudon, France

${ }^{2}$ University College London, Mullard Space Science Laboratory, Holmbury St Mary, Dorking RH5 6NT, UK

${ }^{3}$ Centre National d'Études Spatiales, 18 avenue Edouard Belin, 31401 Toulouse Cedex 9, France

${ }^{4}$ Instituto de Astrofísica de Canarias, 38025 La Laguna, Tenerife, Spain

${ }^{5}$ IMCCE, Observatoire de Paris, CNRS, UPMC, Université Lille 1, 77 avenue Denfert Rochereau, 75014 Paris, France

${ }^{6}$ Cassiopée, Observatoire de la Côte d'Azur, CNRS, BP. 4229, 06304 Nice Cedex 4, France

7 Royal Observatory of Belgium; Ringlaan 3, 1180 Brussels, Belgium

${ }^{8}$ Laboratoire d'Astrophysique de Bordeaux, CNRS, Université Bordeaux 1, 2 rue de l'Observatoire, BP. 89, 33271 Floirac Cedex, France

${ }^{9}$ Astrophysical Institute, University of Liège, allée du 6 Août 17, 4000 Liège, Belgium

10 University of Antwerp, Middelheimlaan, 2020 Antwerpen, Belgium

11 Astrophysikalisches Institut Potsdam, An der Sternwarte 16, 14482 Potsdam, Germany

12 Observatoire Astronomique de l'Université de Genève, 51 chemin des Maillettes, 1290 Sauverny, Switzerland

${ }^{13}$ E2V centre for electronic imaging, Open University, UK

14 GRAAL, CNRS, Université de Montpellier II, place Eugène Bataillon, CC 72, 34095 Montpellier Cedex 05, France

15 Zentrum für Astronomie der Universität Heidelberg, Landessternwarte; Königstuhl 12, 69117 Heidelberg, Germany
} 


\begin{abstract}
During the five years of the mission, the Gaia spectrograph, the Radial Velocity Spectrometer (RVS) will repeatedly survey the celestial sphere down to magnitude $\mathrm{V} \sim 17-18$. This talk presents: (i) the system which is currently developed within the Gaia Data Processing and Analysis Consortium (DPAC) to reduce and calibrate the spectra and to derive the radial and rotational velocities, (ii) the RVS expected performances and (iii) scientific returns.
\end{abstract}

\title{
1 Introduction
}

The on-ground processing of Gaia data is under the responsibility of the Gaia Data Processing and Analysis Consortium (DPAC). The DPAC is structured in 9 Coordination Units (CU), each in charge of one facet of the analysis. The CU6 Spectroscopic Processing is responsible for the first stages of the treatment of the Radial Velocity Spectrometer (RVS) data: from the pre-processing and extraction of the spectra to the derivation of the radial and rotational velocities of the sources observed by the satellite. Section 2 presents the CU6 on-ground processing system. The RVS expected performances are reviewed in Section 3 and some of the RVS science cases are discussed in Section 4.

\section{Processing of RVS data}

\subsection{CU6 structure}

Like the other CUs, CU6 started its activity in 2006. Before Gaia's launch, currently planned late 2012, the work of the CU is devoted to the definition, implementation and test of the RVS ground segment. The CU6 system should be operational before the launch of the satellite in order to be ready to process the commissioning data early 2013. The CU6 processing system is made of about 50 software modules. These are conceived in 12 astronomical institutes $^{1}$ from 5 countries $^{2}$. New or upgraded modules are regularly delivered by the astronomical institutes to the CU6 Data Processing Center (DPC), the Centre National d'Études Spatiales (CNES) in Toulouse. The CU6 DPC integrates the codes in a single environment named SAGA and jointly developed by the Thals company and the CNES. During the operational phase, the data will be processed by the DPC. In CU6, 45 core members work on the development of the ground segment as well as on the calculation and/or observations of auxiliary data required to process the

\footnotetext{
${ }^{1}$ Astrophysikalisches Institut Potsdam, Groupe de Recherche en Astronomie et Astrophysique du Languedoc, Mullard Space Science Laboratory, Observatories of Bordeaux, Geneva, Heidelberg, Nice, Paris, Open University, Royal Observatory of Belgium, Universities of Antwerp, Liège.

${ }^{2}$ Belgium, France, Germany, Switzerland, UK.
} 
RVS spectra (Jasniewicz et al. 2010; Crifo et al. 2010). The CU6 also counts 11 associate members.

\subsection{CU6 tasks}

The CU6 is in charge of the following tasks:

- Pre-process the data: extract from the Gaia Main DataBase (MDB) the information necessary for the CU6 processing. Derive "astrometric" quantities: e.g. date of the observations, location of the sources in the field of view (using codes developed by CU3 astrometric processing).

- Extract the spectra: Transform the telemetry stream into cleaned calibrated spectra that can be analysed.

- Calibrate the spectrograph characteristics: Calibrate the characteristics of the Radial Velocity Spectrometer.

- Perform the single-transit analysis (STA) of the spectra: Derive the radial and rotational velocities for each epoch separately. Issue science alerts for sources with rare behaviour and which require prompt ground-based follow-up.

- Perform the multiple-transit analysis (MTA) of the spectra: Derive the radial and rotational velocities via time series analysis of spectra, radial velocities and correlation peaks. Combine the individual epoch spectra into mission spectra.

- Define the $\mathrm{V}_{R}$ 0-point: Transform the Gaia relative spectroscopic velocities into absolute kinematic velocities (Lindegren \& Dravins 2003; Jasniewicz et al. 2010) The radial and rotational velocities and the spectra are then transferred to other CUs, which combine them with astrometric and spectro-photometric information to characterise the sources (CU8), with special processing for the multiple systems (CU4) and address the case of variable systems (CU7).

\subsection{Spectra extraction}

The Radial Velocity Spectrometer is an integral field spectrograph (see Katz et al. 2004; Katz 2009; Cropper \& Katz 2010, for a presentation of the RVS design). All the light entering its 2 fields of view is dispersed by the grating onto the 12 CCDs of the RVS focal plane. Gaia will be operated at about 1.5 million kilometers from the Earth. At this long distance, the telemetry budget will not allow to transmit continuously the $\sim$ one billion pixels of the Gaia focal plane. Scientifically "interesting" sources will be detected on-board. Only windows around the images of these objects will be read and transmitted to the Earth. When 2 (or several) sources are close, their spectra may overlap and the windows can be (partially) truncated.

The spectra extraction sub-system transforms the stream of RVS windows into cleaned calibrated spectra: it (i) removes the background signal and cosmic ray hits and corrects for the CCD defects, (ii) deblends the overlapped spectra of neighbouring sources, (iii) applies the calibrations and (iv) normalises the spectra to the continuum.

The spectra extraction presents several challenges unique to Gaia data. 
The background below a given spectrum is made of the sum of the spectra of the neighbouring sources. This background is modelled and subtracted from the spectrum of interest.

The cosmic ray hits will damage gradually the CCDs and create traps in the pixels (Cropper \& Katz 2010). These traps will capture and release a part of the signal collected. Some flux will be lost in the observations and the lines will be partially filled and distorted. The impact will be stronger for small signals and will increase along the mission. Rather than correcting the observations for this effect, the synthetic templates used in the subsequent steps of the analysis will be degraded to match the observations.

\subsection{RVS calibration}

Contrary to most ground-based spectrographs, the RVS is not equipped with calibration devices like, e.g. flat field or wavelength calibration lamps. The majority of the RVS characteristics (wavelength, photometric response, Point Spread Function and scattered light) shall be calibrated using the RVS observations themselves. The RVS self-calibration has been inspired by the calibration of the astrometric instrument (Lindegren 2010). To illustrate it, let us consider the calibration of the RVS wavelength dispersion law. First, suitable calibration stars are selected among the RVS observations. For the wavelength calibration, they should display several narrow weak lines. Reference lines (identified, unblended) are then selected. The next step is to solve simultaneously for the dispersion law and for the radial velocities of all the calibration stars. The stars which show significant variations in radial velocity over several transits are considered variable and are rejected as calibrators. At this point, there is a solution for the dispersion law and an estimate of the radial velocity for each calibration star. Yet, dispersion law and radial velocities are mathematically degenerate as a shift in the dispersion law can be compensated by a global shift of all the radial velocities, to still yield a consistent solution. As discussed in Jasniewicz et al. (2010), the degeneracy is removed by using ground-based radial velocity standards.

\subsection{Single transit analysis}

One of the main characteristics of Gaia and of the RVS data is the variety. Variety in signal to noise ratios, from 1000 (for the combined spectra at the end of the mission) to less than 1 (for the single CCD exposure of the faintest targets). Variety in stellar types, as all stages of stellar evolution for the whole stellar mass sequence will be observed. Variety in multiplicity, as single, binaries and higher order multiples will be observed. To cope with this huge variety of cases, 5 software modules are developed to derive the radial and rotational velocities of both single and multiple line systems. Each RVS spectrum will be analysed by the 5 modules. The 5 results will be combined to provide the epoch estimates of the radial velocities as well as the rotational velocities. The single transit analysis sub-system will also provide multiplicity diagnostics. 


\subsection{Multiple transit analysis}

Over the 5 years of the mission, the RVS will observe each source 40 times on average. The time series of spectra, radial velocities and cross-correlation functions (the latter two provided by the STA sub-system) will be analysed and modelled. This will provide variability diagnostics, multiplicity diagnostics, radial velocities for identified single stars, modeled radial velocities for binary systems and mission combined spectra. All this information will be used (together with the astrometry provided by CU3 and the spectro-photometry provided by CU5) by CU4 and CU7 in the detailed analysis of respectively the multiple systems and variable stars and by CU8 in the derivation of the stellar characteristics and in mapping the interstellar extinction.

\subsection{CU6 operations}

- Each day, the last 24 hours of data will analysed with the aim to: (i) check the good health of the instrument, (ii) provide first solutions for the calibrations, radial and rotational velocities and (iii) issue alerts for urgent ground-based follow-up.

- The data will be re-processed from the beginning of the mission by cycles of 6 to 12 months, to iteratively improve the precision of the results, each time with a longer observation baseline and more transits available.

\begin{tabular}{|c|c|c|c|c|c|c|c|c|}
\hline \multicolumn{3}{|c|}{$\begin{array}{l}\text { Table 1. Radial ve- } \\
\text { locity end of mission } \\
\text { performances specifica- } \\
\text { tions. MP: metal-poor: } \\
{[\mathrm{Fe} / \mathrm{H}]=-1.5 \text { dex. }}\end{array}$} & \multicolumn{3}{|c|}{$\begin{array}{l}\text { Table } 2 . \quad \text { Single- } \\
\text { transit and end-of- } \\
\text { mission signal to noise } \\
\text { ratios for a } \mathrm{G} 2 \mathrm{~V} \text { type } \\
\text { star. }\end{array}$} & \multicolumn{3}{|c|}{$\begin{array}{l}\text { Table 3. Limiting magnitudes } \\
\text { and number of targets for the } \\
\text { determination of the different } \\
\text { parameters from RVS spectra. } \\
N \text { is not relevant for } \mathrm{E}_{(B-V)} \text { as } \\
\text { it is not a stellar parameter. }\end{array}$} \\
\hline & $\begin{array}{c}\mathrm{V} \\
\mathrm{mag}\end{array}$ & $\begin{array}{c}\mathrm{V}_{R} \\
\mathrm{~km} \mathrm{~s}^{-1}\end{array}$ & $\begin{array}{c}\mathrm{V} \\
\mathrm{mag}\end{array}$ & $\begin{array}{l}\mathrm{S} / \mathrm{N} \\
\text { tran. }\end{array}$ & $\begin{array}{l}\mathrm{S} / \mathrm{N} \\
\text { miss. }\end{array}$ & Param & $\mathrm{V}$ & $\mathrm{N}$ \\
\hline $\mathrm{B} 1 \mathrm{~V}$ & 7 & 1 & 6 & 150 & 1000 & $\mathrm{~V}_{R}$ & 17 & $150.10^{6}$ \\
\hline B1V & 12 & 15 & 10 & 20 & 150 & $v \sin i$ & 13 & $5.10^{6}$ \\
\hline $\mathrm{G} 2 \mathrm{~V}$ & 13 & 1 & 12 & 8 & 50 & $\mathrm{AP}$ & 13 & $5.10^{6}$ \\
\hline $\mathrm{G} 2 \mathrm{~V}$ & 16.5 & 15 & 14 & 2 & 10 & Abund & 12 & $2.10^{6}$ \\
\hline K1IIIMP & 13.5 & 1 & 16 & & 2 & $\mathrm{E}_{(B-V)}$ & 13 & \\
\hline K1IIIMP & 17 & 15 & & & & & & \\
\hline
\end{tabular}

\section{RVS performances}

Table 1 recalls the requirements for the RVS radial velocity performances, as defined in the Gaia Mission Requirements Document (MRD). Table 2 presents the signal to noise ratio per pixel as a function of magnitude for a $\mathrm{G} 2 \mathrm{~V}$ star for one transit and at the end of the mission. Table 3 presents the RVS limiting magnitude for the determination of stellar and interstellar quantities. Atmospheric parameters (AP), chemical abundances and interstellar reddening will be derived 
by CU8 using all the information available, i.e. astrometric, spectro-photometric and spectroscopic. In particular, for the stellar atmospheric parameters, the RVS spectra will provide useful information down to about magnitude $V=13$, but the spectro-photometry will allow for characterising the stars down to the Gaia limiting magnitude $G=20$. The $862 \mathrm{~nm}$ Diffuse Interstellar Band (DIB) will complement the spectro-photometry as a measure of the interstellar reddening (Munari et al. 2008).

\section{$4 \quad$ RVS scientific returns}

Table 4 presents some of the RVS science cases, the associated tracers and the RVS performances for those tracers. A detailed discussion of the RVS science case can be found in Wilkinson et al. (2005).

Table 4. RVS science cases, associated tracers and performances.

\begin{tabular}{lll} 
Science case & Tracer & Performances \\
\hline Halo streams/merger relics & K2III at $20 \mathrm{kpc}$ & $\sigma_{V r} \leq 10 \mathrm{~km} \mathrm{~s}^{-1}$ \\
Milky-way mass/gravit poten. & RGB tip at $50 \mathrm{kpc}$ & \\
Milky-way mass/gravit poten. & AGB/CH stars at $60 \mathrm{kpc}$ & $\sigma_{V r} \leq 5 \mathrm{~km} \mathrm{~s}^{-1}$ \\
Spiral arms & B stars at $2.5 \mathrm{kpc}$ & \\
Chemical tagging $[\alpha / \mathrm{Fe}]$ & G2V at $250 \mathrm{pc}$ & \\
Chemical tagging $[\alpha / \mathrm{Fe}]$ & K0III at $1.5 \mathrm{kpc}$ & mass determination $^{-1}$ \\
Binaries & 25000 eclipsing SB2 & $\sigma_{V r} \leq 7 \mathrm{~km} \mathrm{~s}^{-1}$ \\
Variable stars & LP class. Cepheids at 20-40 kpc & \\
\hline
\end{tabular}

\section{References}

Crifo, F., Jasniewicz, G., Soubiran, C., et al., 2010, A\&A, in press

Cropper, M., \& Katz, D., 2010, in these proceedings; Gaia: at the frontiers of astrometry, ed. C. Turon, F. Arenou, \& F. Meynadier

Jasniewicz, G., Crifo, F., Soubiran, C., et al., 2010, in these proceedings; Gaia: at the frontiers of astrometry., ed. C. Turon, F. Meynadier \& F. Arenou

Katz, D., 2009, in SF2A-2009: Proceedings of the Annual meeting of the French Society of Astronomy and Astrophysics, ed. M. Heydari-Malayeri, C. Reylé, \& R. Samadi, 57

Katz, D., Munari, U., Cropper, M., et al., 2004, MNRAS, 354, 1223

Lindegren, L., 2010, in IAU Symposium, Vol. 261, "Relativity in Fundamental Astronomy: Dynamics, Reference Frames, and Data Analysis", ed. S.A. Klioner, P.K. Seidelmann, \& M.H. Soffel, 296

Lindegren, L., \& Dravins, D., 2003, A\&A, 401, 1185

Munari, U., Tomasella, L., Fiorucci, M., et al., 2008, A\&A, 488, 969

Wilkinson, M.I., Vallenari, A., Turon, C., et al., 2005, MNRAS, 359, 1306 